\title{
AN EXPLICIT FORMULA FOR THE OPTIMAL GAIN IN THE FULL-INFORMATION PROBLEM OF OWNING A RELATIVELY BEST OBJECT
}

\author{
VLADIMIR V. MAZALOV, ${ }^{*}$ Karelian Research Center of the Russian Academy of Science \\ MITSUSHI TAMAKI, ${ }^{* *}$ Aichi University
}

\begin{abstract}
A full-information version of the secretary problem in which the objective is to maximize the time of possession of a relatively best object is considered both in the case in which only the most recently observed object may be chosen and in the case in which any of the previously observed objects may be chosen. The main purpose of this paper is to obtain, under an optimal rule, both the asymptotic proportional durations when the number of objects tends to infinity, and the expected durations when the number of objects remains finite. The integral expressions for these asymptotic values are derived in both cases: the approximate numerical values are 0.435 in the former (no-recall) case and 0.449 in the latter (recall) case, indicating a surprisingly small difference. The expected values of the optimal stopping times are also obtained from the planar Poisson process analysis.
\end{abstract}

Keywords: Optimal stopping; secretary problem; $\mathrm{e}^{-1}$-law; duration problem; planar Poisson process; monotone subsequence problem

2000 Mathematics Subject Classification: Primary 60G40

\section{Introduction and summary}

A known number of objects, $n$, appear sequentially in random order, and one of them must be chosen. An object that has been rejected cannot be recalled later. In the classical bestchoice problem, sometimes referred to as the secretary problem, the objective is to maximize the probability of choosing the best object. At each time, we observe only the relative rank of the current object with respect to its predecessors. It is well known that the asymptotic value, as $n \rightarrow \infty$, of the optimal rule is $\mathrm{e}^{-1}=0.3678 \cdots$, and that the optimal rule is easily described. Moreover, as the $\mathrm{e}^{-1}$-law (Bruss (1984)) shows, $\mathrm{e}^{-1}$ is the precise lower bound for the value even in the continuous-time model for unknown $n$, if all $n$ objects have independent, identically distributed arrival times. In contrast to the above no-information version of the problem, the informed version is the problem in which the observations are the true values of the objects, assumed to be independent, identically distributed random variables from a known continuous distribution that is taken, without loss of generality, to be the uniform distribution on the interval $[0,1]$. This is often called the full-information version of the problem. In this setting, the asymptotic value (i.e. the probability of choosing the largest of these random variables) increases to $0.5802 \ldots$ (see Gilbert and Mosteller (1966, p. 56)). The classical bestchoice problem was generalized to admit stochastically successful procurement of previously

Received 13 August 2003; revision received 10 October 2005.

* Postal address: Institute of Applied Mathematical Research, Karelian Research Center of the Russian Academy of Science, 11 Pushkinskaya Street, Petrozavodsk, 185610, Russia. Email address: vmazalov@ trc.karelia.ru

** Postal address: Department of Business Administration, Aichi University, Nagoya Campus, 370 Kurozasa, Miyoshi, Nishikamo, Aichi 470-0296, Japan. Email address: tamaki@vega.aichi-u.ac.jp 
rejected objects by Yang (1974) in the no-information version, and by Petruccelli (1982) in the full-information version. Other models of recall with finite memory were considered by Smith and Deely (1975), Rocha (1993), and Tamaki (1986).

Ferguson et al. (1992) extensively considered, as a variation of the secretary problem, the duration problem, where the payoff is the length of time we are in possession of a relatively best object. Thus, the objective of this problem is to find an optimal stopping rule that maximizes the expected duration of owning a relatively best object, or, equivalently, the expected proportional duration (which is defined as (expected duration) $/ n$ ), to make the solution more easily comparable to other problems. In considering the full-information version of the duration problem, Ferguson et al. (1992) were mainly concerned with finding the optimal stopping rule, and left unsolved the problem of obtaining the value attained under an optimal rule. In this paper, we thus attempt to derive the explicit forms of the optimal expected duration for finite $n$ and the asymptotic value of the (optimal expected) proportional duration, both in the case in which only the most recently observed object may be chosen (i.e. the no-recall case), and in the case in which any of the previously observed objects may be chosen (i.e. the recall case).

In Section 2.1, we show that if $c_{1}=2.1198 \cdots$ is a solution to (2.3), then the asymptotic proportional duration in the no-recall case is given by

$$
v_{1}^{*}=\int_{0}^{1} \mathrm{e}^{-c_{1} / u}\left[\int_{0}^{u}\left(\frac{\mathrm{e}^{c_{1} v / u}-1}{v}+\frac{\mathrm{e}^{c_{1} v / u}}{1-v}\right) \mathrm{d} v-1\right] \mathrm{d} u \approx 0.43517 .
$$

Similarly, if $c_{2}=1.3450 \cdots$ is a solution to (2.20), the asymptotic value in the recall case is given, in Section 2.2 , by

$$
v_{2}^{*}=\int_{0}^{1} \int_{0}^{u} \frac{\mathrm{e}^{-c_{2}(1-v) / u}}{1-v} \mathrm{~d} v \mathrm{~d} u \approx 0.44925 .
$$

Remember that the corresponding values in the no-information version are $2 \mathrm{e}^{-2} \approx 0.2707$ in the no-recall case and $\mathrm{e}^{-1} \approx 0.3679$ in the recall case (see Section 2.2 of Ferguson et al. (1992)).

Although the values in (1.1) and (1.2) are obtained as the limiting values of the finite problems in Section 2, they are also derived from the planar Poisson process (PPP) analysis in Section 3, which greatly facilitates the derivation of the asymptotic value for some full-information models (see Gnedin (1996) and Samuels (2004)). The expected values of the optimal stopping times are also obtained.

Interestingly, the value $v_{1}^{*}$ in (1.1) has multiple meanings. The full-information GilbertMosteller problem was modified in different ways by Porosiński (1987) and Petruccelli (1980); Porosiński replaced a fixed $n$ by a random $N$ uniform on $\{1,2, \ldots, n\}$ and independent of the observations, while Petruccelli kept $n$ fixed but allowed the observation of only the sequence of ranges (maximum minus minimum), as well as of whether the current observation is the largest so far. Porosiński (2002) found that these two quite different best-choice problems have identical optimal rules and a common asymptotic value $v_{1}^{*}$. Motivated by Porosiński (2002) and a talk by the second author (given at a conference in Bedlewo, Poland, in July 2002), who reported on the formula (1.1), Samuels (2004) put forward yet another best-choice problem having the same asymptotic value $v_{1}^{*}$, and attempted to explain, using a PPP model, why these extraordinary coincidences occur. Gnedin (2004), inspired by Samuels (2004), considered a more general class of PPPs and gave a resolution to the 'Petruccelli-Porosiński-Samuels paradox' on the coincidence of the asymptotic values. 
Let $N$ again be a random variable uniformly distributed on $\{1,2, \ldots, n\}$. The equivalence between the best-choice problem with $N$ objects and the duration problem without recall, but with a fixed number, $n$, of objects, can be observed not only in the full-information version but also in the no-information version (see Presman and Sonin (1972) for the no-information version of the Porosiński (1987) problem). Although various explanations of this equivalence were given in Samuels (1991), (2004) and Gnedin (2004), it was Gnedin (2005) who established and extended this equivalence in greater generality.

\section{Full-information duration problems}

Let $X_{1}, X_{2}, \ldots, X_{n}$ be independent, identically distributed random variables uniformly distributed on $[0,1]$, where $X_{j}, 1 \leq j \leq n$, denotes the (true) value of the object at the $j$ th stage from last. An object is relatively best if it has the largest value observed so far. We are to select a relatively best object with the view of maximizing the time it stays relatively best. The chosen object is not required to be the best overall. Two cases are distinguished: in the no-recall case, we can choose an object only when a new relatively best object appears, and, in the recall case, we can solicit the current relatively best object wherever it may be. Since $X_{n}$ is observed at time $1, X_{n-1}$ at time 2 , and so on,

$$
L_{k}=\max \left(X_{n}, X_{n-1}, \ldots, X_{k}\right), \quad 1 \leq k \leq n,
$$

represents the largest value to be observed by time $n-k+1$. We denote the state of the process after having observed $X_{n}, X_{n-1}, \ldots, X_{k}$ by a triplet $(x, k, m)$, if $L_{k}=X_{m}=x, k \leq m \leq n$. For a fixed $k$, a serious decision of choice (acceptance or rejection) occurs in state $(x, k, k)$ in the no-recall case, and in state $(x, k, m)$, for all $m$, in the recall case.

Let $T_{k}(x)$ be the time elapsed, conditional on $L_{k}=x$, from time $n-k+1$ until a new relatively best object appears. If no such object appears, let $T_{k}(x)=k$. Then we have

$$
\begin{aligned}
& \mathrm{P}\left\{T_{k}(x)=j\right\}=(1-x) x^{j-1}, \quad 1 \leq j \leq k-1, \\
& \mathrm{P}\left\{T_{k}(x)=k\right\}=x^{k-1},
\end{aligned}
$$

and the expected payoff, when we choose a current relatively best object in state $(x, k, k)$ in the no-recall case, or in state $(x, k, m)$ in the recall case, is defined as

$$
w(x, k)=\mathrm{E}\left(T_{k}(x)\right)=1+x+x^{2}+\cdots+x^{k-1}=\frac{1-x^{k}}{1-x} .
$$

Note that, in the recall case, we do not obtain the whole duration as payoff, but only the portion of the duration initiated at the moment of selection. See Section 3.1 of Ferguson et al. (1992) for more details of the problem considered here.

\subsection{Sampling without recall}

Let $v(x, k)$ denote the optimal expected return attained by leaving state $(x, k+1, k+1)$ and continuing in an optimal manner thereafter. Then the $v(x, k)$ are defined inductively by

$$
v(x, k)=x v(x, k-1)+\int_{x}^{1} \max (w(t, k), v(t, k-1)) \mathrm{d} t, \quad 1 \leq k \leq n, \quad v(x, 0)=0,
$$

and the optimal expected duration $v_{1}^{*}(n)$ is defined as $v(0, n)$. 
After leaving state $(x, k, k)$, if we choose the next relatively best object, if any, we can expect to receive the payoff

$$
\begin{aligned}
u(x, k) & =\sum_{j=1}^{k-1} x^{j-1} \int_{x}^{1} w(t, k-j) \mathrm{d} t \\
& =\sum_{j=1}^{k-1} x^{j-1} \sum_{i=1}^{k-j} \frac{1-x^{i}}{i} .
\end{aligned}
$$

Ferguson et al. (1992) showed that there exists a decision number $b_{k}$ such that the optimal decision in state $(x, k, k)$ is to choose the relatively best object of value $X_{k}=x$ if $x \geq b_{k}$. They also showed that $b_{1}=0$ and that, for $k>1, b_{k}$ is increasing in $k$ and is calculated as the unique root $x \in[0,1]$ of the equation

$$
w(x, k)=u(x, k) .
$$

They also showed that if $b_{k}$ is written as $b_{k}=1-z_{k} / k$, then $z_{k}$ converges, as $k \rightarrow \infty$, to the constant $c_{1}=2.1198 \cdots$ that satisfies the equation

$$
\int_{0}^{1} \mathrm{e}^{-c_{1} v}\left[1-\int_{0}^{1-v} \frac{1-\mathrm{e}^{-c_{1} u}}{u} \mathrm{~d} u\right] \mathrm{d} v=0
$$

Other expressions for (2.2) and (2.3) can be given (see the following lemma) if we introduce the notation $a_{0}=0$ and $a_{k}=\sum_{j=1}^{k} 1 / j, k \geq 1$, and the exponential-integral functions

$$
I(c)=\int_{1}^{\infty} \frac{\mathrm{e}^{-c u}}{u} \mathrm{~d} u, \quad J(c)=\int_{0}^{1} \frac{\mathrm{e}^{c v}-1}{v} \mathrm{~d} v=\sum_{j=1}^{\infty} \frac{c^{j}}{j j !} .
$$

Lemma 2.1. (i) The decision number $b_{k}, k>1$, is a solution of the equation

$$
\sum_{i=0}^{k-1}\left(a_{k-1-i}-a_{i}-1\right) x^{i}=0 .
$$

(ii) The constant $c_{1}$ satisfies the equation

$$
1+J\left(-c_{1}\right)=\mathrm{e}^{-c_{1}}\left[1-J\left(c_{1}\right)\right] .
$$

Proof. (i) Equation (2.4) is immediate from (2.2), since $u(x, k)$ in (2.1) can be written as

$$
\begin{aligned}
u(x, k) & =\sum_{i=1}^{k-1} \sum_{j=1}^{k-i} \frac{x^{i-1}}{j}-\sum_{i=1}^{k-1} \sum_{j=1}^{k-i} \frac{x^{j+i-1}}{j} \\
& =\sum_{i=0}^{k-1} a_{k-1-i} x^{i}-\sum_{i=0}^{k-1} a_{i} x^{i}
\end{aligned}
$$

(ii) Equation (2.5) holds because the left-hand side of (2.3) can be written as follows:

$$
\begin{aligned}
\int_{0}^{1} \mathrm{e}^{-c_{1} v} \mathrm{~d} v-\int_{0}^{1}\left\{\int_{0}^{1-u} \mathrm{e}^{-c_{1} v} \mathrm{~d} v\right\} \frac{1-\mathrm{e}^{-c_{1} u}}{u} \mathrm{~d} u \\
\quad=\frac{1}{c_{1}}\left(1-\mathrm{e}^{-c_{1}}\right)-\frac{1}{c_{1}} \int_{0}^{1}\left\{1-\mathrm{e}^{-c_{1}(1-u)}\right\} \frac{1-\mathrm{e}^{-c_{1} u}}{u} \mathrm{~d} u \\
=\frac{1}{c_{1}}\left[1-\mathrm{e}^{-c_{1}}+J\left(-c_{1}\right)+\mathrm{e}^{-c_{1}} J\left(c_{1}\right)\right]
\end{aligned}
$$


The asymptotic proportional duration is defined as $v_{1}^{*}=\lim _{n \rightarrow \infty} v_{1}^{*}(n) / n$. Explicit expressions for $v_{1}^{*}(n)$ and $v_{1}^{*}$ can be given as follows.

Theorem 2.1. (i) For $n \geq 1$,

$$
v_{1}^{*}(n)=a_{n}+\sum_{k=1}^{n} \sum_{i=0}^{k-1} \frac{1}{n-i}\left(a_{i}-a_{k-1-i}-1\right) b_{k}^{n-i} .
$$

(ii) The asymptotic proportional duration $v_{1}^{*}$ is given as in (1.1) or, equivalently, as

$$
v_{1}^{*}=\left(\mathrm{e}^{c_{1}}-1\right) I\left(c_{1}\right)+\left\{\mathrm{e}^{-c_{1}}-c_{1} I\left(c_{1}\right)\right\} J\left(c_{1}\right) .
$$

Proof. (i) Let $K$ be the time at which the maximum value observed so far initially exceeds the decision number, i.e. $K=\min \left\{k: L_{n+1-k} \geq b_{n+1-k}\right\}$. Also, let $\mathrm{E}\left(D_{n}\right)$ denote the expected duration under an optimal rule. Since we now know the form of the optimal rule, we can obtain $v_{1}^{*}(n)=\mathrm{E}\left(D_{n}\right)$ by conditioning on $\left(K, L_{n+1-K}, L_{n+2-K}\right)$. That is,

$$
\begin{aligned}
\mathrm{E}\left(D_{n}\right)= & \mathrm{E}\left(\mathrm{E}\left(D_{n} \mid K, L_{n+1-K}, L_{n+2-K}\right)\right) \\
= & \sum_{k=1}^{n} \iint_{y \leq x} \mathrm{E}\left(D_{n} \mid K=k, L_{n+1-K}=x, L_{n+2-K}=y\right) \\
& \quad \times p\left(K=k, L_{n+1-K}=x, L_{n+2-K}=y\right) \mathrm{d} x \mathrm{~d} y,
\end{aligned}
$$

where $p\left(K=k, L_{n+1-K}=x, L_{n+2-K}=y\right)$ is the joint probability density of

$$
\left(K, L_{n+1-K}, L_{n+2-K}\right),
$$

and the term corresponding to $k=1$ in the above summation must be interpreted as

$$
\int_{0}^{1} \mathrm{E}\left(D_{n} \mid K=1, L_{n}=x\right) p\left(K=1, L_{n}=x\right) \mathrm{d} x,
$$

because no reference value $L_{n+1}$ is available at time 1 .

It is easy to see that

$$
\mathrm{E}\left(D_{n} \mid K=k, L_{n+1-K}=x, L_{n+2-K}=y\right)= \begin{cases}u(x, n+1-k) & \text { if } y=x, \\ w(x, n+1-k) & \text { if } y<x\end{cases}
$$

and that, for $k \geq 2$,

$$
\begin{aligned}
p(K & \left.=k, L_{n+1-K}=x, L_{n+2-K}=y\right) \\
& = \begin{cases}(k-1) x^{k-1} & \text { if } y=x \text { and } b_{n+1-k} \leq x \leq b_{n+2-k}, \\
(k-1) y^{k-2} & \text { if } y<x \text { and } b_{n+1-k} \leq x, y \leq b_{n+2-k}, \\
0 & \text { otherwise, }\end{cases}
\end{aligned}
$$

with $\mathrm{E}\left(D_{n} \mid K=1, L_{n}=x\right)=w(x, n)$ and

$$
p\left(K=1, L_{n}=x\right)= \begin{cases}1 & \text { if } b_{n} \leq x \\ 0 & \text { if } b_{n}>x\end{cases}
$$


It is a simple matter to check that, for each $n$,

$$
\int_{0}^{1} p\left(K=1, L_{n}=x\right) \mathrm{d} x+\sum_{k=2}^{n} \iint_{y \leq x} p\left(K=k, L_{n+1-K}=x, L_{n+2-K}=y\right) \mathrm{d} x \mathrm{~d} y \equiv 1 .
$$

Substituting (2.10) and (2.11) into (2.9) yields

$$
\begin{aligned}
\mathrm{E}\left(D_{n}\right)= & \int_{b_{n}}^{1} w(x, n) \mathrm{d} x+\sum_{k=2}^{n} \int_{b_{n+1-k}}^{b_{n+2-k}} u(x, n+1-k)(k-1) x^{k-1} \mathrm{~d} x \\
& +\sum_{k=2}^{n}\left\{\int_{b_{n+1-k}}^{b_{n+2-k}} \int_{0}^{x}+\int_{b_{n+2-k}}^{1} \int_{0}^{b_{n+2-k}}\right\} w(x, n+1-k)(k-1) y^{k-2} \mathrm{~d} y \mathrm{~d} x .
\end{aligned}
$$

The first term of (2.12) becomes

$$
\int_{b_{n}}^{1}\left(\sum_{j=1}^{n} x^{j-1}\right) \mathrm{d} x=a_{n}-\sum_{j=1}^{n} \frac{1}{j} b_{n}^{j}
$$

The second term of (2.12), when combined with (2.6), can be transformed into

$$
\begin{aligned}
\sum_{k=1}^{n-1} \int_{b_{k}}^{b_{k+1}} u(x, k)(n-k) x^{n-k} \mathrm{~d} x & \\
= & \sum_{k=1}^{n-1} \int_{b_{k}}^{b_{k+1}}\left\{\sum_{i=0}^{k-1}\left(a_{k-1-i}-a_{i}\right) x^{i}\right\}(n-k) x^{n-k} \mathrm{~d} x \\
= & \sum_{j=2}^{n} \frac{1}{j} \sum_{k=n-j+1}^{n-1}(n-k)\left(a_{n-j}-a_{k-1-n+j}\right)\left(b_{k+1}^{j}-b_{k}^{j}\right) \\
= & \sum_{j=1}^{n} \frac{1}{j} \sum_{k=n-j+1}^{n}\left(a_{n-j}-a_{k-1-n+j}-1\right) b_{k}^{j}+\sum_{j=1}^{n} \frac{1}{j} b_{n+1-j}^{j} \\
& +\sum_{j=2}^{n} \sum_{k=n-j+1}^{n-1} \frac{1}{j-n+k} b_{k+1}^{j}-\sum_{k=1}^{n-1} a_{k} b_{k+1}^{n-k} .
\end{aligned}
$$

The third term of (2.12) can be reduced to

$$
\begin{aligned}
& \sum_{k=1}^{n-1} \int_{b_{k}}^{b_{k+1}} w(x, k) x^{n-k} \mathrm{~d} x+\sum_{k=1}^{n-1} b_{k+1}^{n-k} \int_{b_{k+1}}^{1} w(x, k) \mathrm{d} x \\
& =\sum_{j=1}^{n} \frac{1}{j}\left(b_{n}^{j}-b_{n+1-j}^{j}\right)+\sum_{k=1}^{n-1} a_{k} b_{k+1}^{n-k}-\sum_{j=2}^{n} \sum_{k=n-j+1}^{n-1} \frac{1}{j-n+k} b_{k+1}^{j} .
\end{aligned}
$$

Substituting (2.13)-(2.15) into (2.12) yields (2.7). 
(ii) See Section 1 of Mazalov and Tamaki (2003) for a derivation of (1.1). To transform (1.1) into (2.8), write $c$ instead of $c_{1}$, for simplicity, and rewrite (1.1) as

$$
v_{1}^{*}=I_{\alpha}+I_{\beta}-I_{\gamma}
$$

where

$$
\begin{gathered}
I_{\alpha}:=\int_{0}^{1} \mathrm{e}^{-c / u}\left[\int_{0}^{u} \frac{\mathrm{e}^{c v / u}-1}{v} \mathrm{~d} v\right] \mathrm{d} u, \\
I_{\beta}:=\int_{0}^{1} \mathrm{e}^{-c / u}\left[\int_{0}^{u} \frac{\mathrm{e}^{c v / u}}{1-v} \mathrm{~d} v\right] \mathrm{d} u, \quad \text { and } \quad I_{\gamma}:=\int_{0}^{1} \mathrm{e}^{-c / u} \mathrm{~d} u .
\end{gathered}
$$

Let $x=1 / u$. Then, by integration by parts, $I_{\gamma}$ becomes

$$
I_{\gamma}=\int_{1}^{\infty} \frac{\mathrm{e}^{-c x}}{x^{2}} \mathrm{~d} x=\mathrm{e}^{-c}-c I(c) .
$$

By letting $x=v / u$, we have

$$
\int_{0}^{u} \frac{\mathrm{e}^{c v / u}-1}{v} \mathrm{~d} v=\int_{0}^{1} \frac{\mathrm{e}^{c x}-1}{x} \mathrm{~d} x=J(c),
$$

and so $I_{\alpha}$ becomes

$$
I_{\alpha}=\int_{0}^{1} \mathrm{e}^{-c / u} J(c) \mathrm{d} u=I_{\gamma} J(c)=\left(\mathrm{e}^{-c}-c I(c)\right) J(c) .
$$

Now let $t=1 / u$ and $s=(1-v) / u$ in $I_{\beta}$. Then the Jacobian is $1 / t^{3}$ and, so,

$$
\begin{aligned}
I_{\beta} & =\int_{0}^{1}\left\{\int_{1}^{s+1} \frac{\mathrm{d} t}{t^{2}}\right\} \frac{1}{s} \mathrm{e}^{-c s} \mathrm{~d} s+\int_{1}^{\infty}\left\{\int_{s}^{s+1} \frac{\mathrm{d} t}{t^{2}}\right\} \frac{1}{s} \mathrm{e}^{-c s} \mathrm{~d} s \\
& =\int_{0}^{1} \frac{\mathrm{e}^{-c s}}{1+s} \mathrm{~d} s+\int_{1}^{\infty}\left(\frac{1}{s^{2}}-\frac{1}{s}+\frac{1}{1+s}\right) \mathrm{e}^{-c s} \mathrm{~d} s \\
& =\mathrm{e}^{c} \int_{1}^{2} \frac{1}{x} \mathrm{e}^{-c x} \mathrm{~d} x+\left[\left(\mathrm{e}^{-c}-c I(c)\right)-I(c)+\mathrm{e}^{c} \int_{2}^{\infty} \frac{1}{x} \mathrm{e}^{-c x} \mathrm{~d} x\right] \\
& =\mathrm{e}^{-c}+\left(\mathrm{e}^{c}-c-1\right) I(c) .
\end{aligned}
$$

Thus, substituting (2.17)-(2.19) into (2.16) yields (2.8).

\subsection{Sampling with recall}

Ferguson et al. (1992) showed that, in the recall case, there exists a decision number $b_{k}$ such that the optimal decision in state $(x, k, m)$ is to choose the relatively best object of value $L_{k}=X_{m}=x$, regardless of $m$, if $x \geq b_{k}$. They also showed that $b_{1}=0$ and that, for $k>1$, $b_{k}$ is increasing in $k$ and is calculated as the unique root $x \in[0,1]$ of the equation

$$
\sum_{j=1}^{k-1} \frac{1}{j}\left(1-x^{j}\right)=1
$$


Obviously, the $b_{k}$ here are different from those defined in Section 2.1. It was also shown that, if $b_{k}$ is written as $b_{k}=1-z_{k} / k$, then $z_{k}$ converges, as $k \rightarrow \infty$, to the constant $c_{2}=1.3450 \cdots$ that satisfies the equation

$$
1+J\left(-c_{2}\right)=0 .
$$

Let $v_{2}^{*}(n)$ and $v_{2}^{*}$ respectively denote the optimal expected duration for finite $n$ and the asymptotic proportional duration. Their explicit forms are given as follows.

Theorem 2.2. (i) For $n \geq 2$,

$$
v_{2}^{*}(n)=a_{n}+\sum_{k=2}^{n} \sum_{i=0}^{k-2} \frac{1}{n-i} b_{k}^{n-i} .
$$

(ii) The asymptotic proportional duration $v_{2}^{*}$ is given as in (1.2) or, equivalently, as

$$
v_{2}^{*}=\mathrm{e}^{-c_{2}}+\left(\mathrm{e}^{c_{2}}-c_{2}-1\right) I\left(c_{2}\right) .
$$

Proof. (i) Let $v_{2}^{*}(n)=\mathrm{E}\left(D_{n}\right)$ denote the expected duration under an optimal rule. Since the optimal rule in the recall case is of the same threshold type as in the no-recall case, it is easy to see that (2.9), the formula for $\mathrm{E}\left(D_{n}\right)$, is still valid for the recall case if

$$
\mathrm{E}\left(D_{n} \mid K=k, L_{n+1-K}=x, L_{n+2-K}=y\right)
$$

in (2.10) is replaced by

$$
\mathrm{E}\left(D_{n} \mid K=k, L_{n+1-K}=x, L_{n+2-K}=y\right)=w(x, n+1-k), \quad y \leq x ;
$$

that is to say, (2.12) holds if, in the second term on its right-hand side, $u(x, n+1-k)$ is replaced by $w(x, n+1-k)$. Now,

$$
\begin{aligned}
& \sum_{k=2}^{n} \int_{b_{n+1-k}}^{b_{n+2-k}} w(x, n+1-k)(k-1) x^{k-1} \mathrm{~d} x \\
& \quad=\sum_{k=1}^{n-1} \int_{b_{k}}^{b_{k+1}}\left(\sum_{j=1}^{k} x^{j-1}\right)(n-k) x^{n-k} \mathrm{~d} x \\
& \quad=\sum_{k=1}^{n-1} \sum_{i=0}^{k-1}\left(\frac{n-k}{n-i}\right)\left(b_{k+1}^{n-i}-b_{k}^{n-i}\right) \\
& \quad=\sum_{k=2}^{n} \sum_{i=0}^{k-2} \frac{1}{n-i} b_{k}^{n-i}+\sum_{j=1}^{n} \frac{1}{j} b_{n+1-j}^{j}-\sum_{k=1}^{n-1} b_{k+1}^{n-k}
\end{aligned}
$$

applying (2.13), (2.23), and (2.15) to (2.12) yields

$$
v_{2}^{*}=a_{n}+\sum_{k=2}^{n} \sum_{i=0}^{k-2} \frac{1}{n-i} b_{k}^{n-i}+\sum_{k=1}^{n-1}\left(a_{k}-1\right) b_{k+1}^{n-k}-\sum_{j=2}^{n} \sum_{k=n-j+1}^{n-1} \frac{1}{j-n+k} b_{k+1}^{j} .
$$

Moreover, from the definition of $b_{k+1}$, we have

$$
\sum_{j=2}^{n} \sum_{k=n-j+1}^{n-1} \frac{1}{j-n+k} b_{k+1}^{j}=\sum_{k=1}^{n-1}\left(\sum_{j=1}^{k} \frac{1}{j} b_{k+1}^{j}\right) b_{k+1}^{n-k}=\sum_{k=1}^{n-1}\left(a_{k}-1\right) b_{k+1}^{n-k},
$$

which when combined with (2.24) proves (2.21). 
(ii) Equation (1.2) is a direct consequence of (2.21) because, by setting $b_{k}=1-z_{k} / k$ and then letting $n \rightarrow \infty$, we obtain

$$
\frac{v_{2}^{*}(n)}{n}=\frac{a_{n}}{n}+\frac{1}{n} \sum_{k=2}^{n} \sum_{i=0}^{k-2} \frac{1}{n-i}\left(1-\frac{z_{k}}{k}\right)^{n-i} \longrightarrow 0+\int_{0}^{1} \int_{0}^{u} \frac{1}{1-v}\left(\mathrm{e}^{-c_{2}}\right)^{(1-v) / u} \mathrm{~d} v \mathrm{~d} u .
$$

The transformation of (1.2) into (2.22) follows by analogy with (2.19).

\section{The PPP approach}

As Samuels (2004) and Gnedin (1996), (2004) have shown, the PPP approach turns out to be a powerful tool for studying the limiting behaviors of the full-information models. In this section, we derive by PPP analysis not only the proportional durations given by (2.8) and (2.22), but also the expected values of the optimal stopping times. Following Samuels (2004) (see his Section 9), we employ a planar Poisson process with unit rate on the semi-infinite strip $[0,1] \times[0, \infty)$. This setting inverts the problem, making the 'best' become the 'smallest'. We scan the process from left to right along the strip; the best arrival, second best arrival, etc., have values that are sums of independent, identically distributed mean-1 exponential random variables, and arrive at independent, identically distributed uniform $(0,1)$ times that are independent of these values.

\subsection{Sampling without recall}

Samuels (2004) was mainly concerned with the full-information best-choice problems, but studied the duration problem also and derived (3.7), below, in connection with the Porosiński problem. In the remainder of the paper, we use an argument adapted from his Section 13.2. Let $\mathrm{E}(D(t, y))$ denote the expected duration if we choose the point $(t, y)$ in the PPP, i.e. we stop at time $t$ with a relatively best object of value $y$. Then, from the properties of the PPP, we have

$$
\mathrm{E}(D(t, y))=\int_{0}^{1-t} \mathrm{P}\{D(t, y)>r\} \mathrm{d} r=\int_{0}^{1-t} \mathrm{e}^{-y r} \mathrm{~d} r=\frac{1-\mathrm{e}^{-(1-t) y}}{y} .
$$

If we do not choose this point, but instead choose the point related to the next relatively best object, if any, then, since its value is uniformly distributed on $(0, y)$, we can expect to receive a payoff

$$
\begin{aligned}
q(t, y) & =\int_{0}^{1-t}\left\{\int_{0}^{y} p(t+r, x) \frac{1}{y} \mathrm{~d} x\right\} y \mathrm{e}^{-y r} \mathrm{~d} r \\
& =\int_{0}^{y} \frac{\mathrm{d} x}{x}\left[\frac{1-\mathrm{e}^{-(1-t) y}}{y}-\frac{1}{y-x}\left\{\mathrm{e}^{-(1-t) x}-\mathrm{e}^{-(1-t) y}\right\}\right],
\end{aligned}
$$

where we have written $p(s, x)$ for $\mathrm{E}(D(s, x))$. We now write $p(t, y)=q(t, y)$ using (3.1) and (3.2), which yield

$$
1-\mathrm{e}^{-(1-t) y}=\int_{0}^{y} \frac{\mathrm{d} x}{x}\left[1-\mathrm{e}^{-(1-t) y}-\frac{y}{y-x}\left\{\mathrm{e}^{-(1-t) x}-\mathrm{e}^{-(1-t) y}\right\}\right]
$$

or, equivalently,

$$
1-\mathrm{e}^{-c}=\int_{0}^{1} \frac{\mathrm{d} v}{v}\left[1-\mathrm{e}^{-c}-\frac{1}{1-v}\left(\mathrm{e}^{-c v}-\mathrm{e}^{-c}\right)\right]
$$

where $c=(1-t) y$. 
Since the right-hand side of (3.3) can be expressed as

$$
\begin{aligned}
\int_{0}^{1} \mathrm{~d} v & {\left[\frac{1-\mathrm{e}^{-c}}{v}-\left(\frac{1}{v}+\frac{1}{1-v}\right)\left(\mathrm{e}^{-c v}-\mathrm{e}^{-c}\right)\right] } \\
= & -\int_{0}^{1} \frac{\mathrm{e}^{-c v}-1}{v} \mathrm{~d} v-\mathrm{e}^{-c} \int_{0}^{1} \frac{\mathrm{e}^{c(1-v)}-1}{1-v} \mathrm{~d} v \\
= & -\left[J(-c)+\mathrm{e}^{-c} J(c)\right],
\end{aligned}
$$

having $p(t, y)=q(t, y)$ is equivalent to having

$$
1+J(-c)=\mathrm{e}^{-c}[1-J(c)] .
$$

It follows from (2.5) that $c=c_{1}$ satisfies (3.4). Moreover, since $p(t, y) \geq q(t, y)$ implies that $p\left(t^{\prime}, y^{\prime}\right) \geq q\left(t^{\prime}, y^{\prime}\right)$ for $t^{\prime}>t$ and $y^{\prime}<y$, we conclude that the optimal rule stops with the first relatively best object, if any, that lies under the curve $y=c_{1} /(1-t)$.

The following argument, referred to as the 'forward-looking argument' in Samuels (2004), leads to the calculation of the duration $\mathrm{E}\left(D_{c}\right)$ expected under the optimal stopping rule specified by the threshold curve $y=c /(1-t)$.

Let

$T=$ the arrival time of the first (left-most) point that lies below the curve $y=c /(1-t)$.

$S=$ the time at which the value of the best (lowest) above-threshold arrival is equal to the threshold.

Then $S$ and $T$ are independent random variables, and have the following respective densities:

$$
f_{S}(s)=\frac{c s}{(1-s)^{c+2}} \mathrm{e}^{-c s /(1-s)} \quad \text { and } \quad f_{T}(t)=c(1-t)^{c-1} .
$$

Samuels (2004) gave the result

$$
\begin{aligned}
\mathrm{E}\left(D_{c}\right)= & \int_{0}^{1} \int_{0}^{t} p\left(s, \frac{c}{1-s}\right) f_{S}(s) f_{T}(t) \mathrm{d} s \mathrm{~d} t \\
& +\int_{0}^{1} \int_{0}^{s}\left[\frac{1-t}{c} \int_{0}^{c /(1-t)} p(t, y) \mathrm{d} y\right] f_{T}(t) f_{S}(s) \mathrm{d} t \mathrm{~d} s,
\end{aligned}
$$

and showed that this simplifies to

$$
\mathrm{E}\left(D_{c}\right)=\mathrm{e}^{-c}-1-J(-c)+\left\{(c+1)\left(\mathrm{e}^{c}-1\right)+c \mathrm{e}^{c} J(-c)\right\} I(c) .
$$

Theorem 3.1. (i) The optimal expected duration becomes

$$
\mathrm{E}\left(D_{c_{1}}\right)=\left(\mathrm{e}^{c_{1}}-1\right) I\left(c_{1}\right)+\left\{\mathrm{e}^{-c_{1}}-c_{1} I\left(c_{1}\right)\right\} J\left(c_{1}\right),
$$

which coincides with $v_{1}^{*}$ in (2.9).

(ii) Let $\tau_{1}$ denote the optimal stopping time. Its expected value is given by

$$
\mathrm{E}\left(\tau_{1}\right)=\mathrm{e}^{-c_{1}}+\left(\mathrm{e}^{c_{1}}-c_{1}-1\right) I\left(c_{1}\right) \approx 0.336 .
$$

Moreover, the probability, say $\mathrm{P}\left\{\tau_{1}=1\right\}$, that the optimal stopping rule comes to a dead end without choosing an object is given by

$$
\mathrm{P}\left\{\tau_{1}=1\right\}=\mathrm{e}^{-c_{1}}-c_{1} I\left(c_{1}\right) \approx 0.032 .
$$

Proof. (i) Equation (3.7) is immediate from (3.6) because $c=c_{1}$ satisfies (3.4). 
(ii) For $c=c_{1}$, let $S$ be defined as before and let $T^{\prime}$ be defined as a random variable, depending on $S$, that denotes the arrival time of the first (left-most) point that lies below the curve

$$
y=\min \left(\frac{c}{1-t}, \frac{c}{1-S}\right),
$$

if such a point exists, and which equals 1 otherwise. Since the number of points in any region of the PPP is Poisson with parameter equal to the region's area, the density of $T^{\prime}$ conditional on $S$ is given by

$$
\begin{aligned}
f_{T^{\prime} \mid S}(t) & =-\frac{\mathrm{d}}{\mathrm{d} t} \mathrm{P}\left\{T^{\prime}>t \mid S\right\} \\
& =-\frac{\mathrm{d}}{\mathrm{d} t} \exp \left(-\int_{0}^{t} \min \left(\frac{c}{1-r}, \frac{c}{1-S}\right) \mathrm{d} r\right) \\
& = \begin{cases}c(1-t)^{c-1} & \text { if } t \leq S, \\
c(1-S)^{c-1} \mathrm{e}^{-c(t-S) /(1-S)} & \text { if } t>S .\end{cases}
\end{aligned}
$$

Moreover, the conditional probability that no object is chosen until the end becomes

$$
\mathrm{P}\left\{T^{\prime}=1 \mid S\right\}=\exp \left(-\left[\int_{0}^{S} \frac{c}{1-t} \mathrm{~d} t+\int_{S}^{1} \frac{c}{1-S} \mathrm{~d} t\right]\right)=\mathrm{e}^{-c}(1-S)^{c} .
$$

Straightforward calculations using (3.10) and (3.11) yield the conditional expectation of $T^{\prime}$ given $S$ to be

$$
\begin{aligned}
\mathrm{E}\left(T^{\prime} \mid S\right) & =\int_{0}^{1} \mathrm{P}\left\{T^{\prime}>t \mid S\right\} \mathrm{d} t \\
& =\frac{1}{c+1}+\frac{1-(c+1) \mathrm{e}^{-c}}{c(c+1)}(1-S)^{c+1} .
\end{aligned}
$$

Since $\tau_{1}$ is distributed as is $T^{\prime}$, from (3.12) and (3.5) we have

$$
\begin{aligned}
\mathrm{E}\left(\tau_{1}\right) & =\mathrm{E}\left(\mathrm{E}\left(T^{\prime} \mid S\right)\right) \\
& =\int_{0}^{1}\left[\frac{1}{c+1}+\frac{1-(c+1) \mathrm{e}^{-c}}{c(c+1)}(1-s)^{c+1}\right] \frac{c s}{(1-s)^{c+2}} \mathrm{e}^{-c s /(1-s)} \mathrm{d} s \\
& =\frac{1}{c+1}+\frac{\mathrm{e}^{c}-c-1}{c+1} \int_{0}^{1} \frac{s}{1-s} \mathrm{e}^{-c /(1-s)} \mathrm{d} s \\
& =\frac{1}{c+1}+\frac{\mathrm{e}^{c}-c-1}{c+1} \int_{1}^{\infty}\left(\frac{1}{u}-\frac{1}{u^{2}}\right) \mathrm{e}^{-c u} \mathrm{~d} u \quad\left(\text { where } u=\frac{1}{1-s}\right) \\
& =\frac{1}{c+1}+\frac{\mathrm{e}^{c}-c-1}{c+1}\left[I(c)-\left\{\mathrm{e}^{-c}-c I(c)\right\}\right] \quad(\text { from }(2.17)) \\
& =\mathrm{e}^{-c}+\left(\mathrm{e}^{c}-c-1\right) I(c),
\end{aligned}
$$

which proves (3.8). Equation (3.9) can be obtained from $\int_{0}^{1} \mathrm{P}\left\{T^{\prime}=1 \mid S\right\} f_{S}(s) \mathrm{d} s$.

Remark 3.1. It is easy to see that, for any $c>0$, (3.8) and (3.9) hold if $\tau_{1}$ is replaced by the stopping rule which chooses the first relatively best object that lies under the curve $y=c /(1-t)$. Moreover, the probability of such a rule making the best choice can be shown to be given by the 
right-hand side of (3.7) (see Samuels (1991) or Section 10.3 of Samuels (2004)). The optimal rule for the Gilbert-Mosteller best-choice problem is of this form with $c=c_{3}=0.8043 \cdots$, which is the solution to $J(c)=1$. For this value of $c$, (3.7) and (3.8) are identical, with common value $0.5801 \cdots$, while (3.9) becomes $0.1995 \cdots$, as obtained by Gnedin (1996).

\subsection{Sampling with recall}

Denote by $(t, y)$ the state in which the relatively best object observed up to time $t$ has value $y$. If we choose the relatively best in this state, then the expected duration is $p(t, y)=$ $\left\{1-\mathrm{e}^{-(1-t) y}\right\} / y$, as given in Section 3.1, while, if we continue for a small additional time $\Delta t$ and then stop (i.e. stop with the new relatively best object, if one appears, and otherwise solicit the current such object), then the expected duration is given by

$$
q(t, y, \Delta t)=y \Delta t \int_{0}^{y} p(t+\Delta t, x) \frac{1}{y} \mathrm{~d} x+(1-y \Delta t) p(t+\Delta t, y)+o(\Delta t),
$$

because $y \Delta t$ is the probability that a new relatively best object appears during the interval $(t, t+\Delta t)$. Thus, if

$$
p(t, y) \geq q(t, y, \Delta t)
$$

stopping immediately at time $t$ is at least as good as waiting an additional small time $\Delta t$ and then stopping. Since

$$
p(t+\Delta t, y)=p(t, y)-\mathrm{e}^{-(1-t) y} \Delta t+o(\Delta t),
$$

(3.13) can be written as

$$
p(t, y) \geq \Delta t \int_{0}^{y} p(t+\Delta t, x) \mathrm{d} x+p(t, y)-\left\{y p(t, y)+\mathrm{e}^{-(1-t) y}\right\} \Delta t+o(\Delta t)
$$

or, equivalently,

$$
0 \geq\left[\int_{0}^{y} p(t+\Delta t, x) \mathrm{d} x-y p(t, y)-\mathrm{e}^{-(1-t) y}\right] \Delta t+o(\Delta t)
$$

or

$$
0 \geq \int_{0}^{y} p(t, x) \mathrm{d} x-y p(t, y)-\mathrm{e}^{-(1-t) y},
$$

by dividing both sides by $\Delta t$ and then letting $\Delta t \rightarrow 0$. If we set $c=(1-t) y,(3.14)$ reduces to

$$
\int_{0}^{1} \frac{1-\mathrm{e}^{-c x}}{x} \mathrm{~d} x \leq 1
$$

or, equivalently,

$$
-J(-c) \leq 1
$$

The set of states $(t, y)$ that satisfy (3.15) can be regarded as an 'infinitesimal look-ahead stopping region'. Since (3.15) shows that the problem is monotone, the infinitesimal look-ahead stopping region turns out to be an optimal stopping region (see Ross (1971)). 
Theorem 3.2. (i) The optimal expected duration becomes

$$
\mathrm{E}\left(D_{c_{2}}\right)=\mathrm{e}^{-c_{2}}+\left(\mathrm{e}^{c_{2}}-c_{2}-1\right) I\left(c_{2}\right),
$$

which coincides with $v_{2}^{*}$ in (2.22).

(ii) Let $\tau_{2}$ denote the optimal stopping time. Its expected value is given by

$$
\mathrm{E}\left(\tau_{2}\right)=1-c_{2} \mathrm{e}^{c_{2}} I\left(c_{2}\right) \approx 0.348
$$

Proof. (i) It is easy to see that the locus of points $(t, y)$ that satisfy (3.15) with equality constitutes the curve $y=c_{2} /(1-t)$, by (2.20). Thus, (3.16) follows from (3.6).

(ii) For $c=c_{2}$, let $S$ be defined as before and let $T^{\prime \prime}$ be defined as a random variable, depending on $S$, that denotes the arrival time of the first (left-most) point that lies below the curve $y=$ $c /(1-t)$, if such a point appears before $S$, and which equals $S$ otherwise.

As in the proof of part (ii) of Theorem 3.1, we have

$$
\begin{gathered}
f_{T^{\prime \prime} \mid S}(t)=c(1-t)^{c-1}, \quad t<S, \\
\mathrm{P}\left\{T^{\prime \prime}=S \mid S\right\}=\exp \left(-\int_{0}^{S} \frac{c}{1-t} \mathrm{~d} t\right)=(1-S)^{c} .
\end{gathered}
$$

Hence, the conditional expectation is given by

$$
\begin{aligned}
\mathrm{E}\left(T^{\prime \prime} \mid S\right) & =\int_{0}^{S} t f_{T^{\prime \prime} \mid S}(t) \mathrm{d} t+S \mathrm{P}\left\{T^{\prime \prime}=S \mid S\right\} \\
& =\frac{1}{c+1}\left[1-(1-S)^{c+1}\right] .
\end{aligned}
$$

Since $\tau_{2}$ is distributed as is $T^{\prime \prime}$, from (3.17) and (3.5) we have

$$
\begin{aligned}
\mathrm{E}\left(\tau_{2}\right) & =\mathrm{E}\left(\mathrm{E}\left(T^{\prime \prime} \mid S\right)\right) \\
& =\frac{1}{c+1} \int_{0}^{1}\left[1-(1-s)^{c+1}\right] \frac{c s}{(1-s)^{c+2}} \mathrm{e}^{-c s /(1-s)} \mathrm{d} s \\
& =\frac{1}{c+1}\left[1-c \mathrm{e}^{c}\left\{(1+c) I(c)-\mathrm{e}^{-c}\right\}\right] \\
& =1-c \mathrm{e}^{c} I(c),
\end{aligned}
$$

which completes the proof.

Remark 3.2. It is interesting to note that the value $c_{3}$ appears in a problem considered by Bruss and Delbaen (2001), namely that of sequentially selecting a monotone subsequence of maximal expected length in the case in which a sequence of independent, identically distributed random variables appear, over a given horizon $t$, according to a Poisson process with unit rate. They showed that, for small $t$, i.e. $t \leq c_{3}$, the optimal rule selects the relatively best observation successively (see their Lemma 2.3 and Remark 2.1). This is not a pure coincidence, because interrecord times in a Poisson process and record frequencies are strongly related, but we have not studied this in more detail. The authors thank a referee for pointing out this coincidence. 
Remark 3.3. Define, as a function of $c, K(c):=\mathrm{e}^{-c}+\left(\mathrm{e}^{c}-c-1\right) I(c)$. This function appears occasionally in this paper, and represents different quantities of interest depending on the value of $c$. These are summarized as follows.

- $K\left(c_{1}\right)$ is the expected value of the optimal stopping time for the full-information duration problem without recall.

- $K\left(c_{2}\right)$ is the optimal expected duration for the full-information duration problem with recall.

- $K\left(c_{3}\right)$ has two meanings in the full-information best-choice problem: on the one hand it is the optimal probability of choosing the best value, and on the other hand it is the expected value of the optimal stopping time. See also Gnedin and Sakaguchi (1992).

\section{Acknowledgements}

The authors would like to thank Professor S. Samuels for his stimulating talks and discussions at the 2002 Bedlewo conference and also for his invaluable comments and kind suggestions on the first draft of this paper. The transformation in the proof of Theorem 2.1(ii) was demonstrated by Z. Porosiński at the Bedlewo conference. The authors would like to thank Professor Porosiński for his kind suggestion that his result be included in this paper. They are also grateful to the referee for his very detailed criticisms and thoughtful comments, which led to considerable improvements. The first author is grateful to the Japan Society for the Promotion of Science (grant no. L01530) for financial support, and the second author is grateful to Aichi University (grant no. C-117). The second author is also grateful to Professor K. Szajowski for his encouragement.

\section{References}

Bruss, F. T. (1984). A unified approach to a class of best choice problems with an unknown number of options. Ann. Prob. 12, 882-889.

Bruss, F. T. AND Delbaen, F. (2001). Optimal rules for the sequential selection of monotone subsequences of maximum expected length. Stoch. Process. Appl. 96, 313-342.

Ferguson, T. S., Hardwick, J. P. and Tamaki, M. (1992). Maximizing the duration of owning a relatively best object. In Strategies for Sequential Search and Selection in Real Time (Amherst, MA, 1990; Contemp. Math. 125), American Mathematical Society, Providence, RI, pp. 35-57.

Gilbert, J. P. And Mosteller, F. (1966). Recognizing the maximum of a sequence. J. Amer. Statist. Assoc. 61, $35-73$.

Gnedin, A. V. (1996). On the full information best-choice problem. J. Appl. Prob. 33, 678-687.

Gnedin, A. V. (2004). Best choice from the planar Poisson process. Stoch. Process. Appl. 111, 317-354.

Gnedin, A. V. (2005). Objectives in the best-choice problems. Sequent. Anal. 24, 177-188.

GNedin, A. V. AND SAKAGUCHI, M. (1992). On a best choice problem related to the Poisson process. In Strategies for Sequential Search and Selection in Real Time (Amherst, MA, 1990; Contemp. Math. 125), American Mathematical Society, Providence, RI, pp. 59-64.

Mazalov, V. V. And Tamaki, M. (2003). Explicit solutions to the duration problem. Aichi Keiei Ronsyu 147, 69-92.

Petruccelli, J. D. (1980). On a best choice problem with partial information. Ann. Statist. 8, 1171-1174.

Petruccelli, J. D. (1982). Full-information best-choice problems with recall of observations and uncertainty of selection depending on the observation. Adv. Appl. Prob. 14, 340-358.

Porosiński, Z. (1987). The full-information best choice problem with a random number of observations. Stoch. Process. Appl. 24, 293-307.

PorosińsKi, Z. (2002). On best choice problems having similar solutions. Statist. Prob. Lett. 56, 321-327.

Presman, E. L. And Sonin, I. M. (1972). The best choice problem for a random number of objects. Theory Prob. Appl. 17, 657-668.

Rocha, A. L. (1993). The infinite secretary problem with recall. Ann. Prob. 21, 898-916.

Ross, S. M. (1971). Infinitesimal look-ahead stopping rules. Ann. Math. Statist. 42, 297-303. 
Samuels, S. M. (1991). Secretary problems. In Handbook of Sequential Analysis, eds B. K. Ghosh and P. K. Sen, Marcel Dekker, New York, pp. 381-405.

Samuels, S. M. (2004). Why do these quite different best-choice problems have the same solutions? Adv. Appl. Prob. 36, 398-416.

Smith, M. H. And Deely, J. J. (1975). A secretary problem with finite memory. J. Amer. Statist. Assoc. 70, $357-361$.

TAMAKI, M. (1986). A full-information best-choice problem with finite memory. J. Appl. Prob. 23, 718-735.

YANG, M. C. K. (1974). Recognizing the maximum of a random sequence based on relative rank with backward solicitation. J. Appl. Prob. 11, 504-512. 